\title{
Energy Efficient Coverage Optimization in Wireless Sensor Networks based on Genetic Algorithm
}

\author{
Ali Norouzi *, Ahmet Sertbas \\ Department of Computer Engineering, Istanbul University, Avcilar, Turkey
}

Copyright (C) 2015 by authors, all rights reserved. Authors agree that this article remains permanently open access under the terms of the Creative Commons Attribution License 4.0 International License

\begin{abstract}
There has been a great deal of attention paid to Genetic Algorithm (GA). The algorithm, as a methodology, is a multi objective methodology which can be used in different fields such as self-organizing wireless sensor network. The technique examines the applied parameters and at the same time takes into consideration the fitness function by the way of or considering the whole operational modes in produced feasible states. Majority of the GA implementations in clustering algorithm only deal with optimization of few parameters including coverage and energy consumption with noticeable effect on network quality. Keeping network coverage can be modeled as mathematical programming problem which is featured with heavy load of computation. On the other hand, wireless sensor networks (WSNs) can be of dynamic nature, if so it needs to have proper reaction to events; so that slightest management decision may lead to considerable problems on the quality of the network. This problem is dealt with in this study through a hybrid method in MATLAB with the help of Genetic Algorithm toolbox and custom codes. The optimum solution was obtained by mathematical algorithm that conforms to all the mentioned parameters.
\end{abstract}

Keywords Genetic Algorithm, Wireless Sensor Network, Coverage Maintenance, Energy Consumption, Network Optimization

\section{Introduction}

Wireless Sensor Networks (WSN) consist of many low-power, low-cost sensors with limited computational and communication capability. The sensors are easily deployable in WSN setting for specific functions. The cost of wireless platforms is decreasing while theirs functionality increases. This trend promises a widespread use of the wireless system for different purposes such as health monitoring or military sensing. Like other networks, WSNs have inspired enormous number of researchers all over the world $[1,2]$.

A crucial issue or WSNs is the coverage, which is narrowly pertinent to energy saving, connectivity, network reconfiguration, network lifetime, and so on. Nodes in the present study are deployed for random sensor field. Thus, a key problem is coverage as it has effects on monitoring and tracking object.

In general, coverage is discussable from two points of views; the best and the worst observable cases. Under the former approach, the goal is to spot the area of higher observation change and to determine the best support and guidance regions. However, under the latter case, the purpose is to spot the areas with lower observation change and to detect the blind spots. In several cases, a preferred distribution of sensors in the target field is hardly achievable as manual deployment is almost impossible and it may be affected by uncontrollable factors such as wind and obstacles $[3,4]$. Fig. 1 shows the general scheme of network coverage in WSN [5].

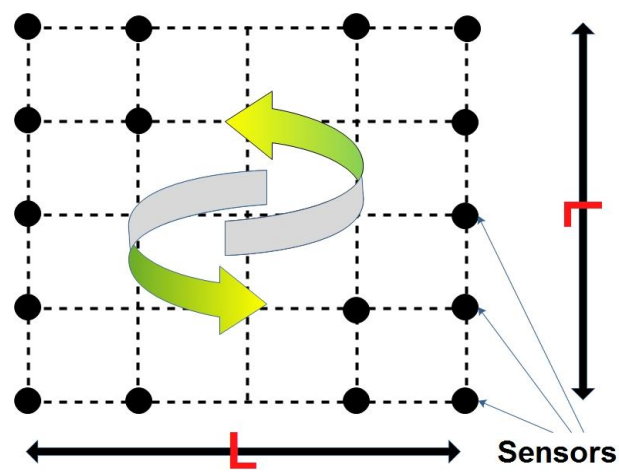

Figure 1. Network Coverage concept in WSN

Network longevity is the most important challenge of WSN because nodes with limited power-energy batteries are in charge of aggregating and transferring monitored data to the base station. These tasks are energy consuming and replacing or recharging the batteries is infeasible.

Few algorithms and protocols have tried to keep balance energy consumption and at the same time keeping a satisfied coverage as a way to solve the issue of lifetime.

Thus, it is essential to study the issues of distributing the sensors evenly, so that the sensors use equal amount of 
energy with acceptable energy coverage. At any rate, the problem of coverage cannot be avoided.

As pointed out, WSN coverage is used to the coverage space of sensors with considerable effect on the performance. This results in considerable decrease of the power consumption.

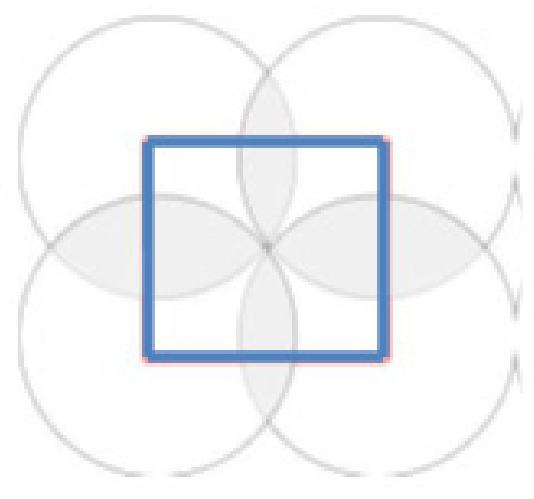

Figure 2. coverage problem in WSN

The area covered by the sensor may also have overlaps (Fig.2). By decreasing the overlaps, the network can cover larger space with fewer sensors. The issues of shared space covered by the sensors in a network are the "coverage problem" [6]. Another problem to be concerned with is the area remained uncovered between the covered areas (Fig.3). The spaces are technically called "holes" and as the network cannot collect information about the holes, minimizing the hole is vital.

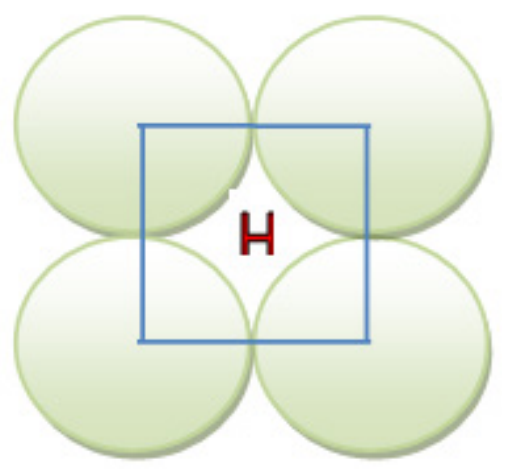

Figure 3. Coverage hole in WSN

Although, failure of a few nodes may not halt the overall network functions, which can have a deep influence over the optimum network connectivity depending the protocol used by the network [7].

Cluster based protocols are used more commonly as the optimum energy consumption is achieved by the clustered nodes. For nodes under cluster architecture, different groups of nodes are formed based on the duties assigned to the network and purposes of cluster. Many nodes members are assigned with a head node known as cluster head. The cluster head transfers the collected data from the member of the cluster to the sink. The cluster heads can schedule their group based on specific Time Division Multiple Access (TDMA).
In spite of the fact that there are several algorithms introduced $[8,9]$, concentration on a specific issue leads to a complicated design.

One of the most promising heuristic methods to deal with the problems of optimization is genetic algorithm (GA). The algorithm is based on inherent selection which also plays a role in biological evolution. The GA modifies a group of individuals frequently and randomly to act as parents and use them to produce the next generation. Afterward, the population evolves to achieve an optimal solution by the way of successive generation. To put it another word, the present study mainly tries to answer the problem of coverage in WSNs, while minimizing the overlapping influence. Simulations were done in MATLAB.

In what follows, section II reviews the literature of WMSN and on network coverage. Section III deals with the basic concepts including terminology and the hypotheses needed in the proposed method. Also solution of the problem of coverage by the proposed algorithm is discussed in section III followed by section IV on evaluation of performance and results of simulation. Section $\mathrm{V}$ is the conclusion.

\section{Related Works}

In majority of recent studies, sensor nodes are static and plenty of extra nodes are installed to reach the preferred level of coverage. This may cause heavy costs, while there is no guaranteed coverage by random distribution.

There have been several algorithms proposed to design an optimum WSN, each of which has concentrated on a specific issue and led to a complex design. This section explains some of previous studies on the problems of network coverage optimization.

One of the pioneering works in the field was conducted by Ref. [10]. He proposed a blanket coverage method that developed a statistical arrangement of sensor nodes, which eventuated in an extra coverage.

Reference [8] Proposed a GA-based method which used a repulsive behavior to scatter the nodes over the desired area. The authors worked on a complete model network in which only offline planning was required for further development. Reference [11] proposed an interactive method, based on GA, to reach an optimal solution for energy consumption, schedule of transmission, and so on.

Another method was introduced in Ref. [12] to determine and add to multimedia coverage of each sensor. The method is designed to automatically position the sensor. As the method requires, the coverage ratio of a specific number of nodes can be obtained and effectiveness of the method is reduced by decreasing of the number of nodes in the method.

A novel classification for WSNs based on 3D geometrical figures was introduced by Ref.[2]. To obtain the covered volume by the sensors, they proposed an equation under the title of "volume in the spherical coordination." Apparently, the classification is practical in places where the space is not in the surface [2]. 
Reference [13] simplified the complex coverage problem though a step by step process. With the use of mathematical modeling, theoretical analysis and formula deducting, classical geometric theories and via mathematical induction, the analysis formula of minimum number of nodes was deduced theoretically under circumstances of entire and seamless coverage in WSN [13].

The problem of mobility of sensor networks was dealt by Ref. [3] in which some part of the sensors could change their locations. When a sensor spots coverage holes, it changes its position following specific policies. To have less cost of sensor mobility, they offered a density mobility scheme (DMS) that may improve coverage of the system through displacing the nodes to the nearest hole found. Under the DMS, a mobile sensor can change its position to a sparse area only when its neighbor nodes cover majority of the sensing area [3].

Another novel modeling of the problem of coverage in wireless camera-bases sensor networks was introduced by genetic algorithm to reduce overlapping effect as a solution to coverage problem [14].

Theoretical analysis of the problem has been employed to increase coverage of an area up to a maximum level with reduced overlapping by the cameras.

Reference[15]introduced a GA based interactive approached for Mobile Sensor Networks. In this work, the fitness function was used to obtain the suitable direction of node locomotion, keeping in mind either coverage of the target area or estimation of the optimum energy consumption.

\section{Proposed Algorithm}

The present study uses genetic algorithm to achieve better solution than that the other methods do. The GA includes the general steps as following.

1. To generate randomly an initial population $\mathrm{M}(0)$.

2. To compute and keep the fitness $u(m)$ for each individual " $\mathrm{m}$ " in the present population $\mathrm{M}(\mathrm{t})$.

3. To define selection probabilities $\mathrm{p}(\mathrm{m})$ for every member " $m$ " in $M(t)$ so that $p(m)$ is relative to $u(m)$.

4. To generate $\mathrm{M}(\mathrm{t}+1)$ by choosing the individuals probabilistically out of $\mathrm{M}(\mathrm{t})$ to form new generation by employing crossover and mutation.

5. Repeat step 2 until satisfying solution is achieved.

GA is usually used to solve the problems featured with wide search space in which accuracy does not matter. As our method calls, the maximum area of monitoring is fully covered by a minimum active sensor.

We assume a WSN with many number of small battery-driven sensor nodes installed in the target field. The sensor nodes sense the environment for information such as temperature on periodical bases. This section introduces the network definition and assumption.

Definition 1: The sensing range of a sensor is a geographical area in which any event may occur.

Definition 2: the sensing neighbor: a set of sensors positioned in sensing range.

Definition 3: A WSN is homogenous when all the sensors have equal sensing range, communication range, and initial energy. Otherwise, it is heterogeneous [4].

Definition 4: Fitness function is to evaluate the goodness of each solution; i.e. chromosome.

Taking into account actual WSN application, the present study considers hypothetical parameters $\mathrm{A}, \mathrm{B}$, and $\mathrm{C}$ for $2 \mathrm{D}$ fields; the parameters help us to follow more practical approach. They are commonly used by researches in the field [16]. Thus, we have three kinds of sensors to observe specific objects. To simplify the problem, it is assumed that the spatial variability of $\mathrm{A}, \mathrm{B}$, and $\mathrm{C}$ are pictured as the density of sensors in area unit in which the objects are monitored in the form of $\rho_{A}<<\rho_{B}<<\rho_{C}$. This concept both deals with general and specific aspects of special objective networks. The simulation parameters are shown in Table 1.

Table 1. Simulation parameters

\begin{tabular}{|c|c|}
\hline Area $(\mathrm{N})$ & $100 * 100$ \\
\hline Number of Sensors(N) & 200 \\
\hline Initial Energy & $2 \mathrm{~J}$ \\
\hline Node energy & $50 \mathrm{nj} / \mathrm{bit}$ \\
\hline Base Station distance & $200 \mathrm{~m}$ \\
\hline Packet Size & $200 \mathrm{bits}$ \\
\hline Sensing range & $15 \mathrm{~m}$ \\
\hline
\end{tabular}

Assume squared Euclidean filed with length 1 subdivided into equal area so that any interested subarea is monitored by sensors located at the vicinal intersection lines. This trick is adopted by many researchers as grid based wireless sensor network layout .

The sensors are small, limited-powered, with certain-transmission range, and sensing-mode selection node, which can choose one of the three operating modes on the basis of its capability and condition status. Since density of parameter $\mathrm{A}$ is the lowest, it has highest transmission range and accordingly $\mathrm{C}$ has lowest range. In this work, clustering solution was adopted to achieve optimum energy consumption as the cluster consists of one certain adjoining sensor with same operating mode - cluster-in-charge. Every cluster communicates with the Base Station (BS) or the sink through multi-hop.

A multi-objective algorithm deals with optimization of two important parameters of energy consumption and coverage problem. Thus, the problem was divided into two sub-problems. The strategy is consisted of:

1. Finding the solution for coverage and power consumption issues by finding minimum number of nodes which are needed to provide coverage for the whole environment with the use of local search and genetic algorithm.

2. To make sure that clusters-in-charge and member 
nodes of a cluster are connected. To this end Kruskal algorithm was used.

A novel algorithm was adopted to propose some of possible optimum network topologies that also minimize constraints such as operational energy, number of unconnected nodes, and overlap of cluster-in-charge errors.

Part of genetic algorithm formula which is actually an improved version of Nakmura formula was used to propose an appropriate fitness function [17]:

$A$ is the given monitoring area, $S$ denotes set of sensor nodes, $D$ stands for set of demanded points, $A^{d}$ is set of sensors monitoring demanded areas, $N C$ is penalty cost of no coverage of demand point, $A E$ is turning energy on, $P C$ denotes penalty cost of path from every node to $B S$ (obtained by Dijekstra's algorithm during a pre-processing phase) and it is assigned to every node to distinguish expensive nodes and the model variables; $X_{i j}$ is 1 (when node $i$ covers demand point $j$ ) and 0 otherwise, $y_{i}$ is 1 (when nodes $i$ is active and 0 otherwise), $h_{j}$ is 1 (when the demand point $j$ is not covered).The variables of the model are:

$X_{i j}=1$ when node ' $\mathrm{i}$ ' covers demand point $\mathrm{j}$ and 0 otherwise

$y_{i}=1$ when nodes ' $\mathrm{i}$ ' is active and 0 otherwise

$h_{j}=1$ when demand point ' $\mathrm{j}$ ' is not covered.

The model can be formulated as:

$$
\min \sum_{i \in s}\left(A E_{i}+P C_{i}\right) \times y_{i}+\sum_{j \in D} N C_{j} \times h_{j}
$$

Subject to:

$$
\begin{gathered}
\sum_{i j}\left(x_{i j}+h_{j}\right) \geq 1, \forall j \in D \& \forall i j \in A^{d} \\
x_{i j} \leq y_{i}, \forall i \in S \& \forall i j \in A^{d} \\
0 \leq x_{i j} \leq 1, \forall i j \in A^{d}, h_{j} \geq 0, \forall j \in D \\
y \in\{0,1\},\{x, h\} \in \mathfrak{R}
\end{gathered}
$$

The above formula minimizes the number of required active node, which leads to increase in network energy as well as the number of uncovered interested area. Constraints 2 and 3 indicate each demanded point monitored by a sensor or remained uncovered and imposes that only active node can sense respectively.

We improve our Fitness Function (FF) of algorithm through considering penalty cost of overlapping cluster-in-charge errors and energy consumption represented by OPCE and EC respectively as follows [18].:

$$
\begin{aligned}
& F F=\min \left(\text { Usage }_{-} \text {Cost }+ \text { Penalty_Cost }\right) \\
& \text { Usage_Cost }=\sum_{i \in s}\left(A E_{i}+P C_{i}+E C\right) \times y_{i} \\
& \text { Penalty_Cost }=\sum_{j \in M} \sum_{k \in D}\left(N C_{j k}+O P C E_{j k}\right) \times h_{k}
\end{aligned}
$$

$$
\text { Subject to: } \quad M \in\{A, B, C\}
$$

$E C$ is numerically measured and it depends upon the mode of sensor network. Obviously, since the sensor node in "A" mode has high communication range it consumes main portion of the energy followed by mode $\mathrm{B}$ and $\mathrm{C}$ in order of regarding energy consumption. In what follows, it is assumed that $\mathrm{A}$ mode consumes four times the power that is used by $\mathrm{C}$ and $\mathrm{B}$ mode consumes two times more than $\mathrm{C}$ mode. $\mathrm{EC}$ is given by:

$$
E C=\frac{4 n_{A}+2 n_{B}+n_{C}}{\sum_{i \in S} n_{i}}
$$

OPCE in the fitness function computes wasted energy for monitoring errors of overlapping cluster-in-charge.

\section{Evaluation and Results}

Plenty of optimum solutions are obtained by genetic based algorithm, though connectivity of the nodes is not taken into account. This presents outflow of collected data toward the BS. Kruskal algorithm was utilized to examine connectivity of network in the 2nd part of ECEP. The process proposed is comprised of four steps:

The network is assumed as graph $\mathrm{G}$ where an edge exists between vertices ' $x$ ' and ' $y$ ' in graph $G$ when the maximum communication range between two particular nodes ' $x$ ' and ' $y$ ' exceeds the distance between ' $x$ ' and ' $y$ '. By introducing the Kruskal algorithm, a minimum spanning tree (MST) is achieved so that a shorter path between each two vertices for routing aggregated data is achieved.

The connectivity with specific shortest paths is achieved when the number of MST tree edges is the same as the number of vertices -1; otherwise, inactive nodes are activated (this explains shorter transmission range than communication distance for some nodes). Kruskal technique is used on newly activated and disconnected nodes. This results in formation of new lightest tree.

The shortest path between each disconnected node to the BS is obtained and the internal sensor nodes of the paths are added to the set $\mathrm{E}$.

Any newly activated node not listed in E is turned deactivated. This helps preservation of network energy while the quality is the same.

Finally, one or two network typology(s) were developed based on the range of transmission of nodes and position of sensor node. The network(s) that realized maximum network coverage are characterized with optimum coverage and energy usage.

To implement the new approach, a square L*L field was used in which the subdivided areas are about equal. Each node is positioned at the intersection of subareas and they can take values 1 : inactive $=00 ; 2$ : mode "A" active $=01 ; 3$ : 
mode " $\mathrm{B}$ " active $=10$; and 4: mode " $\mathrm{C}$ " active $=11$. The network encoding technique is pictured in Fig. 4.

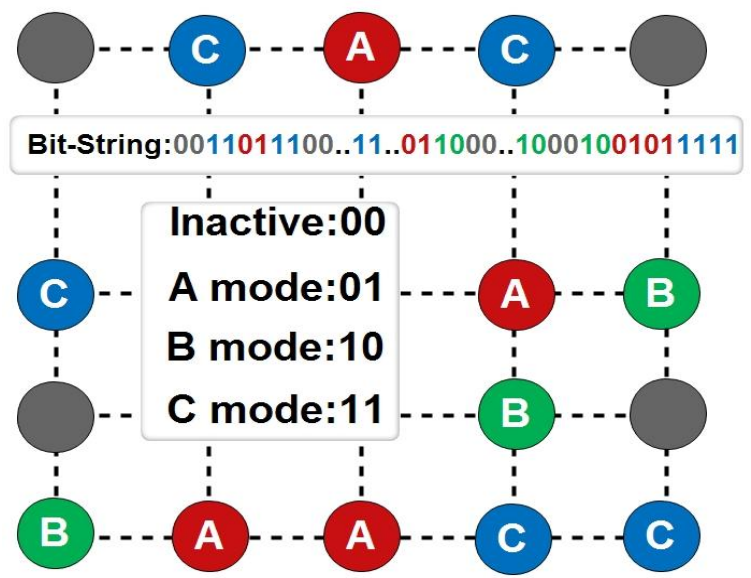

Figure 4. Network with represented encoding

The setting of the whole nodes in network can be represented by gen and the ordered set of gen makes a specific chromosome. In this way, for a network with $\mathrm{L}$ nodes, encoding is $2 . \mathrm{L} 2$ bits, (L.2 gens that need 2 bits to explain the mode of corresponding node). Genetic algorithm technique encompasses a set of chromosomes which are known as the population that are improved by generation process [18].

To put it another way, the algorithm copies the nature as it has input data, and the primary population that are randomly obtained. Following generation process, the developed population or the result represents the optimum solution regarding main problem. All of the improvement carried out by generation process cover crossover, scoring, selection, and mutation functions. By crossover we refer to a production function with specific rate which mates two difference chromosomes to develop a new population. Among the many crossover methods, single point was adopted in this work. Scoring or assigning by fitness function is the key of genetic algorithm that is influenced by the problem [19]..

At this part, the algorithm assigns a weight to any chromosome according to its content. That is, every chromosome stands for a solution that is developed through iterations. Higher fitness value is obtained by the better chromosome, which survives to the next generation. The fitness function is mainly designed based on the problem and the researchers mainly concentrate on representing intelligent fitness function to differentiate qualified individuals. Under the selection process, the better chromosome to create the new population with mutation technique is adopted so that some specific chromosomes are entered to the new generation.

In this paper, population size, productive function, mutation rate were adopted 400 , single point, and 0.5 respectively. Table 2 shows the GA parameters used to simulate the environment. The candidate chromosomes can be chosen randomly because this selection does not affect the final results, i.e., any candidate individuals will tend toward the optimum solution. The number of iterations is constant at 100 .

Table 2. GA parameter Values

\begin{tabular}{|c|c|}
\hline Number of candidate individuals & 400 \\
\hline Length of Chromosome & 20 \\
\hline Crossover Rate & .5 \\
\hline Mutation Rate & .2 \\
\hline Iteration & 100 \\
\hline
\end{tabular}

Because of stochastic bases of GA, repetitive runs of the algorithm eventuated in different solutions with different performances. Thus, average of the results of runs was reported. By using WSN simulator, appx. of the implemented proposed algorithm achieved $100 \%$ coverage on the designated area. Power consumption, number of active nodes, and live packet over time are listed in Table 1. The starting number of 18 packets delivered to the BS was increased to 83 packets after second 01.18 .

Table 2. Observed values in the early times of network

\begin{tabular}{|c|c|c|c|}
\hline Time (Nanosecond) & Power & $\begin{array}{c}\text { Active Sensors / } \\
35\end{array}$ & $\begin{array}{c}\text { Live } \\
\text { Packets }\end{array}$ \\
\hline $00: 35.090$ & 20718 & 27 & 18 \\
\hline $00: 49.330$ & 15332 & 27 & 60 \\
\hline $01: 08.107$ & 8031 & 20 & 53 \\
\hline $01: 18.182$ & 6739 & 18 & 83 \\
\hline $01: 36.719$ & 5333 & 14 & 30 \\
\hline $01: 48.626$ & 4368 & 12 & 36 \\
\hline $02: 02.956$ & 3354 & 10 & 38 \\
\hline $02: 09.666$ & 3123 & 8 & 38 \\
\hline $02: 18.048$ & 2897 & 8 & 47 \\
\hline $02: 46.709$ & 2032 & 7 & 54 \\
\hline $02: 55.352$ & 1860 & 6 & 52 \\
\hline
\end{tabular}

Table 3 indicates the last time the network which died in 5:13:781. In addition, the number of live packets gradually decreases as the number of active sensors approaches 0 .

Table 3. Observed values in the last times of network

\begin{tabular}{|c|c|c|c|}
\hline $\begin{array}{c}\text { Time } \\
\text { (Nanosecond) }\end{array}$ & Power & $\begin{array}{c}\text { Active } \\
\text { Sensors/35 }\end{array}$ & Live Packets \\
\hline $03: 22.821$ & 1096 & 5 & 87 \\
\hline $03: 45.804$ & 616 & 4 & 89 \\
\hline $03: 58.382$ & 421 & 3 & 75 \\
\hline $04: 15.196$ & 307 & 2 & 33 \\
\hline $04: 39.882$ & 122 & 1 & 25 \\
\hline $05: 13.781$ & 0 & 0 & 0 \\
\hline
\end{tabular}

Fig.5 represents effect of number of iterations of genetic algorithm on the network lifetime. Iteration 50 and 57 indicate that increasing individuals does not always lead to more optimal solution. In other words, satisfying stop 
criterion is sufficient to obtain optimal solution.

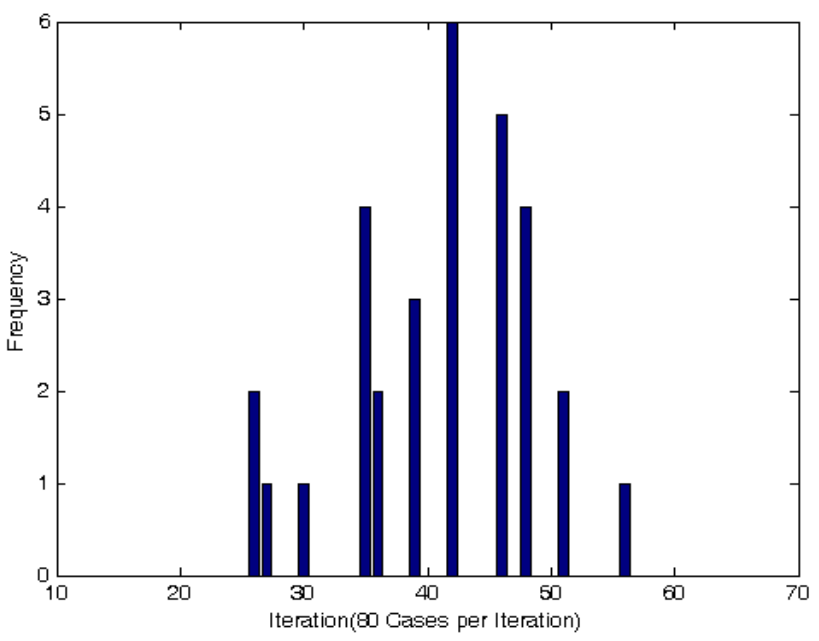

Figure 5. Network lifetime in specified scale

\section{Conclusions}

GA is a one of the commonly adopted solution when the search must be done on a wide area and accuracy of the results is not much of a concern. Based on the proposed method, the widest feasible area was covered using a minimum number of active sensors. A hybrid method was proposed in this study to deal with the main problems of WSN (e.g. coverage and energy consumption) to this end, two genetic algorithms and Kurskal techniques were used. Within the GA based part both the coverage and optimization of energy consumption were under consideration. The main achievement of the present study was demonstration of the capacity of general algorithm to find the solution of coverage problem in WSNs. Three types of sensors with high, medium, and low ranges of transmission were adopted for simulation; the sensors were used to monitor a grid based environment. Results of simulation showed merits of using large number of low power sensors for communication over using less number of sensors with high energy consumption.

\section{REFERENCES}

[1] H. Kaschel, J. Mardones, Gustavo Quezada, "Safety in wireless sensor networks: Types of attacks and solutions, Studies in Informatics and Control, vol. 22 (3), pp. 323-329, ISSN 1220-1766, 2013, [Online]. Available: http://sic.ici.ro/sic2013_3/art09.php

[2] N. Attarzadeh, A. Barati, A. Movaghar, "A new method for coverage in wireless sensor networks," in Proc. Dependable, Autonomic and Secure Computing, 2009. DASC'09. Eighth IEEE International, China, 2009, pp.369-372, doi:10.1109/DASC.2009.142, [Online]. Available: http://ieeexplore.ieee.org/stamp/stamp.jsp?tp=\&arnumber $=5$ 380395 \&isnumber $=5380134$
[3] E. Zhao, Y. Lv, "A density mobility scheme for improving coverage in wireless sensor networks," in Proc. Web Information Systems and Mining, , China, 2009, pp.724-727, doi: 10.1109/WISM.2009.149, [Online]. Available: http://ieeexplore.ieee.org/stamp/stamp.jsp?tp=\&arnumber $=5$ 369335\&isnumber $=5367999$

[4] A. Norouzi, A. Sertbas, "Efficiency analysis and comparative performance evaluation of routing protocols in mobile Ad Hoc networks", Studies in Informatics and Control, vol. 21 (2), pp. 209-220, 2012, ISSN 1220-1766, [Online]. Available: http://sic.ici.ro/sic2012_2/art11.php

[5] F.P. Quintão, F.G. Nakamura, G.R, Mateus, "A hybrid approach to solve the coverage and connectivity problem in wireless sensor networks," In Proc. 4th European Workshop on Meta-heuristics: Design and Evaluation of Advanced Hybrid Meta-heuristics, United Kingdom, 2004, [Online]. Available:

http://www.pessoal.utfpr.edu.br/ewille/Homepage/resource0 3/FredPaper.pdf

[6] M. K. watfa, and S. Commuri, "A coverage algorithm in 3D wireless sensor networks", in Proc. 1st International Symposium on Wireless Pervasive Computing, Thailand, 2006, pp. 16-18, doi: 10.1109/ISWPC.2006.1613560, [Online]. Available:

http://ieeexplore.ieee.org/stamp/stamp.jsp?tp=\&arnumber=1 613560\&isnumber $=33870$

[7] C. H. Kaschel, B. L. Sanchez, F. J. G. Mardones, C. G. Quezada C., "Design and construction of link quality and localization protocol algorithms at WSN over IEEE802.15.4 physical protocol ", Studies in Informatics and Control, vol. 20 (3), pp. 285-292, 2011, ISSN 1220-1766, , 2011, [Online]. Available: http://sic.ici.ro/sic2011_3/art09.php

[8] A. Howard, M. Mataríc, G. Sukhatme, "Mobile sensor network deployment using potential fields: a distributed, scalable solution to the area coverage problem," in Proc. International Symposium on Distributed Autonomous Robotics Systems, Japan, 2002, pp.299-308. [Online]. Available:

http://link.springer.com/chapter/10.1007\%2F978-4-431-659 41-9 30

[9] A. Mollanejad, L. M. Khanli, M. Zeynali, “ DBSR: Dynamic base station Repositioning using Genetic algorithm in wireless sensor network", IJCSI International Journal of Computer Science ,vol. 7, Issue 2, No 2,pp. 24-28, March 2010. [Online]. Available:

http://arxiv.org/ftp/arxiv/papers/1004/1004.3268.pdf

[10] D Gage, "Command control for many-robot systems," Unmanned Systems Magazine, vol. 10, no. 4, pp. 28-34, 1992.

[11] S. Hussain, A. W. Matin, and O. Islam, "Genetic algorithm for energy efficient clusters in wireless sensor networks," in Proc. $4^{\text {th }}$ International Conference on Information Technology: New Generations, USA, 2007, pp. 147-154, doi: 10.1109/ITNG.2007.97, [Online].Available:

$\mathrm{http} / /$ ieeexplore.ieee.org/stamp/stamp.jsp?tp=\&arnumber $=4$ $151674 \&$ isnumber $=4151645$

[12] N.Tezcan, and W. Wang, "Self-orienting wirelsess multimdia sensor networks for occlusion-free viewpoints", Computer Networks, Volume 52, Issue 13, pp.2558-2567, ISSN 1389-1286, [Online]. Available:

http://dx.doi.org/10.1016/j.comnet.2008.05.014. 
[13] Xueqing Wang, Fayi Sun, Xiangsong Kong, "Research on optimal coverage problem of wireless sensor networks", in Proc. International Conference on Communications and Mobile Computing, Yunnan, 2009, pp. 548-552, DOI 10.1109/CMC.2009.231, [Online]. Available:

$\mathrm{http}$ ://ieeexplore.ieee.org/stamp/stamp.jsp?tp=\&arnumber $=4$ 797057\&isnumber $=4796933$

[14] Habibizad Navin , B. Asadi, S. Hassan pour, M. Mirnia, "Solving coverage problem in wireless camera-based sensor networks by using genetic algorithm", in Proc. IEEE International Conference on Computational Intelligence and Communication Systems, India, 2010, pp 226-230 , DOI 10.1109/CICN.2010.54.Available:

$\mathrm{http}: / /$ ieeexplore.ieee.org/stamp/stamp.jsp?tp=\&arnumber $=5$ 701968\&isnumber $=5701925$

[15] A. Norouzi, F. S. Babamir, A. H. Zaim, "An interactive genetic algorithm for mobile sensor networks", Studies in Informatics and Control, vol. 22 (2), pp. 213-218, 2013, ISSN 1220-1766, [Online]. Available: http://sic.ici.ro/sic2013_2/art11.php

[16] A. P. Bhondekar, R. Vig, M. L. Singla, C Ghanshyam, P. Kapur, "Genetic algorithm based node placement methodology for wireless sensor network", in Proc.
International Multi Conference of Engineers and Computer Scientists, Hong Kong, 2009, vol. I, ISBN: 978-988-17012-2-0 [Online]. Available:

http://www.iaeng.org/publication/IMECS2009/IMECS2009 pp106-112.pdf

[17] F.G.Nakamura, "Planejamento din.amico para controle de cobertura e conectividade em redes de sensores sem fio planas." Master's thesis, Universidade Federal de Minas Gerais (in Portuguese), PP 45-78, 2003.

[18] Ali Norouzi and Abdul Halim Zaim, "Genetic Algorithm Application in Optimization of wireless sensor networks (Accepted for publication 19 November 2013) " , The Scientific World Journal, Hindawi publishing cooperation , to be published. Available : http://www.hindawi.com/journals/tswj/aip/286575/

[19] A. Norouzi, F. Babamir and A. Zaim, "A New Clustering Protocol for Wireless Sensor Networks Using Genetic Algorithm Approach," Wireless Sensor Network, Vol. 3 No. 11, 2011, pp. 362-370. doi: 10.4236/wsn.2011.311042.

[20] Akyildiz, I. F. and Vuran, M. C. (2010) Front Matter, in Wireless Sensor Networks, John Wiley \& Sons, Ltd, Chichester, UK. doi: 10.1002/9780470515181. 